\title{
Relación entre el desequilibrio de músculos flexores y extensores de rodilla y las lesiones musculares en atletas de la Selección Mexicana de Taekwondo
}

\author{
José Luis Reyes Salazar, PSS. Ft. \\ Ivonne Stefanie Curiel Sigler, PSS. Ft \\ Gustavo Argenis Hernández Segura, M.I.M \\ Universidad Autónoma de Querétaro, México
}

Doi:10.19044/esj.2021.v17n10p64

Submitted: 24 November 2020

Accepted: 12 February 2021

Published: 31 March 2021
Copyright 2021 Author(s)

Under Creative Commons BY-NC-ND

4.0 OPEN ACCESS

Cite As:

Reyes Salazar J.L., Curiel Sigler I.S. \& Hernández Segura G.A. (2021). Relación entre el desequilibrio de músculos flexores y extensores de rodilla y las lesiones musculares en atletas de la Selección Mexicana de Taekwondo. European Scientific Journal, ESJ, 17(10), 64. https://doi.org/10.19044/esj.2021.v17n10p64

\section{Resumen}

Introducción: El taekwondo es un arte marcial moderna y actualmente uno de los deportes de combate olímpico más populares, se caracteriza por técnicas de patadas y altos niveles de fuerza en la musculatura extensora y flexora de rodilla. El alto índice de lesiones musculares y la suspensión de la actividad deportiva que conlleva han ocasionado que aumente cada vez más el interés en la atención pero sobre todo en la prevención de la lesión. Objetivo: Identificar la relación entre el desequilibrio y las lesiones musculares, específicamente de los músculos isquiotibiales, en una población de atletas de taekwondo de alto rendimiento. Metodología: Se trata de un estudio transversal, descriptivo y correlacional. El estudio se realizó con 15 atletas de la Selección Mexicana de Taekwondo siendo el principal criterio de exclusión el que no estuvieran en proceso para juegos olímpicos 2021, las variables estudiadas fueron el desequilibrio muscular medido a través de dinamometría isocinética y el número de lesiones musculares en isquiotibiales en el último año. El estudio fue aprobado por el comité de bioética de la Universidad Autónoma de Querétaro y está apegado al código de Nuremberg, la declaración de Helsinki y la Ley General de salud. Resultados: Para las variables cuantitativas se utilizó la prueba exacta de Fisher para datos 
categóricos y examinar la asociación. El 47\% de la muestra reportó un desequilibrio muscular significativo y al menos una lesión muscular en isquiotibiales en el último año del mismo lado que el desequilibrio $(p=.007)$ y destacó la extremidad izquierda con el mayor grado de desequilibrio con un 57\%. Conclusión: La alta incidencia de lesiones musculares en el ámbito deportivo y específicamente en esta población, debe ser atendida de manera integral por un equipo multidisciplinario mediante la aplicación de test y evaluaciones regulares, que permitan identificar focos de alarma que puedan predisponer al atleta a una lesión deportiva en el futuro.

Palabras clave: Fuerza muscular, cuádriceps, músculos isquiotibiales

\title{
Relationship Between Knee Flexor and Extensor Muscle Imbalance and Muscle Injuries in Athletes of the Mexican Taekwondo Team
}

\author{
José Luis Reyes Salazar, PSS. Ft. \\ Ivonne Stefanie Curiel Sigler, PSS. Ft \\ Gustavo Argenis Hernández Segura, M.I.M \\ Universidad Autónoma de Querétaro, México
}

\section{Abstract}

Introduction: Taekwondo is a modern martial art and currently one of the most popular Olympic combat sports, it is characterized by kicking techniques and high levels of strength in the knee flexor and extensor muscles. The high rate of muscle injuries and the suspension of sports activity that it entails have caused an increasing interest in care but especially in injury prevention. Objective: To identify the relationship between muscle imbalance and injuries, specifically of the hamstrings, in a population of highperformance taekwondo athletes. Methodology: This is a cross-sectional, descriptive and correlational study. The study was carried out with 15 athletes from the Mexican Taekwondo National Team, the main exclusion criterion being that they were not in process for the 2021 Olympic games, the variables studied were muscle imbalance measured through isokinetic dynamometry and the number of muscle injuries in hamstrings in the past year. The study was approved by the bioethics committee of the Autonomous University of Querétaro and is attached to the Nuremberg Code, the Declaration of Helsinki, and the General Health Law. Results: For quantitative variables, Fisher's exact test was used for categorical data and the association was examined. $47 \%$ of the sample reported a significant muscle imbalance and at least one hamstring 
muscle injury in the last year on the same side as the imbalance $(\mathrm{p}=.007)$ and highlighted the left limb with the highest degree of imbalance with 57\%. Conclusion: The high incidence of muscle injuries in the sports field and specifically in this population must be comprehensively addressed by a multidisciplinary team through the application of regular tests and evaluations, which block the identification of sources of alarm that may predispose the athlete to a future sports injury.

Keywords: Muscle strength, quadriceps muscle, hamstring muscle

\section{Introduction}

El taekwondo se ha convertido en un deporte de combate olímpico y en uno de los deportes de contacto más practicados en la actualidad, se estima que aproximadamente 80 millones de sujetos lo practican en todo el mundo (Fortina, Mangano, Carta y Carulli, 2017). Se caracteriza por combates breves en los cuales predominan principalmente técnicas que requieren a las extremidades inferiores para realizar patadas potentes, en otras palabras, el taekwondo destaca por altos niveles de fuerza en la musculatura de los miembros inferiores para asegurar la ejecución eficaz de las diversas técnicas durante el combate.

Está altamente comprobado que la práctica deportiva induce efectos benéficos en la salud a corto y a largo plazo, sin embargo, esta representa al mismo tiempo un riesgo potencial de lesiones musculoesqueléticas para los atletas (Fortina et al., 2017). En un estudio epidemiológico de taekwondo que reporta dos periodos olímpicos (1997-2000 y 2001-2004) se señalan a las lesiones musculares en el muslo como el cuarto puesto de incidencia (Altarriba-Bartes et al., 2014); del mismo modo, de Hoyo (2013) indica que el bíceps femoral en su porción larga y al tendón proximal del semimembranoso son el lugar donde habitualmente se localizan las lesiones musculares.

El origen de estas lesiones puede suceder por diversos mecanismos, por un traumatismo directo en la zona afectada o como sucede más comúnmente, por una elongación. Este último, puede tener lugar en la ejecución del gesto deportivo, durante una contracción muscular excéntrica y cuando la tensión muscular es máxima (Balius y Pedret, 2013). El alto índice de lesiones musculares y la suspensión de la actividad deportiva que conllevan han ocasionado que aumente cada vez más el interés en la atención pero sobre todo en su prevención (Pruna, 2015).

Desde la antigüedad, en el ámbito de la medicina deportiva, ha sido fundamental el estudio del rendimiento muscular, para lo cual, numerosos autores han optado por la evaluación isocinética, por brindar información más precisa, fiable y manejable. La dinamometría isocinética como sistema informático y robótico de evaluación se utiliza para procesar los datos 
cuantitativos obtenidos de la capacidad muscular (Loeza, Fritzeler y Barrios, 2014) al ejecutar un movimiento contra resistencia en un rango determinado de movimiento y una resistencia constante y programable (Martínez et al., 2014). Dicha evaluación suele utilizarse de forma comparativa entre un segmento corporal y su homónimo opuesto o entre sí mismo (Ayala, Sainz, Croix y Santoja, 2012). Los valores cuantitativos registrados tras la evaluación suelen obtenerse en términos de magnitudes físicas de fuerza, potencia y trabajo, expresados en newton-metro (Nm), watt (W) y joule (J) respectivamente (Loeza et al., 2014; Martínez et al., 2014).

Actualmente esta herramienta es utilizada también con frecuencia para evaluar el equilibrio muscular al comparar la fuerza generada entre grupos musculares. Para su medición se han propuesto diversos protocolos de evaluación y parámetros tanto de velocidad como de tipo de contracción utilizada, entre los que destacan, los índices convencional y funcional, cada uno de ellos con características específicas que los diferencian entre sí. En el índice convencional se utiliza la contracción concéntrica para ambos grupos musculares, mientras que en el índice funcional se utiliza la contracción concéntrica para el cuádriceps y la contracción excéntrica para los isquiotibiales (Ayala etal., 2012). Así mismo, las velocidades utilizadas en ambos casos suelen utilizarse entre 40 a $60^{\circ}$ s para evaluar la fuerza y de 60 a $120 \%$ s para medir el trabajo (Cruz-Medina et al., 2008).

Maly, Mala, Bujnovsky, Hank y Zahalca (2019) refieren que la diferencias de fuerza entre grupos de músculos aparecen en atletas jóvenes y pueden generar a largo plazo desequilibrios en la adaptación de los tejidos. Dicho equilibrio constituye un parámetro importante en el desempeño deportivo de un atleta (Martínez et al., 2014). Así mismo, un desequilibrio importante se ha asociado con un incremento en la probabilidad de sufrir desgarros de la musculatura isquiotibial (Santos, Pedrinelli, Rubio, Guilherme y D' Andrea, 2016; van Dyk et al., 2016).

A pesar de la vasta evidencia científica publicada acerca del desequilibrio muscular como factor de riesgo, existen muy pocos estudios dedicados al taekwondo y la prevención de lesiones en este deporte. Por ello, el objetivo de esta investigación es determinar la relación del desequilibrio de fuerzas entre la musculatura flexora y extensora de la rodilla en el desarrollo de lesiones musculares de isquiotibiales específicos para la población de deportistas de taekwondo de alto rendimiento en México. Todo esto sería útil en la prevención de este tipo de lesiones, estableciendo factores predisponentes que pueden ser atendidos de forma temprana, disminuyendo la incidencia de lesiones musculares y mejorando el desempeño deportivo. 


\section{Metodología}

Se realizó un estudio de tipo transversal, descriptivo y correlacional, con una muestra de 15 de atletas de la Selección Mexicana de Taekwondo.

El protocolo de investigación fue revisado y aprobado por el Subcomité de investigación de la Licenciatura en Fisioterapia de la Facultad de Enfermería de la Universidad Autónoma de Querétaro, posteriormente, el Comité de Bioética de la misma facultad e institución. El desequilibrio en las extremidades inferiores fue evaluado mediante dinamometría isocinética, así mismo, las lesiones musculares previas serán obtenidas mediante el expediente médico de cada atleta.

a) Parámetros

Para calcular el porcentaje de desequilibrio muscular se utilizó el índice convencional, el cual resulta de la división del pico máximo de torque de los flexores entre el pico máximo de torque de los extensores de rodilla. A pesar de haber poco consenso del valor normal de la proporción isquiotibiales/cuádriceps, se considera 0.60 como el valor con mayor aceptación (Tlatoa, 2014; Martínez et al., 2014).

b) Participantes

Se invitó a participar en el estudio a todos aquellos deportistas activos de la Selección Mexicana de Taekwondo. Como criterios de inclusión esenciales se consideró que no estuvieran en proceso de preparación para juegos olímpicos 2020 y que estuvieran sanos de cualquier condición que pudiera alterar los resultados de la prueba. Todos los participantes fueron mayores de 18 años con distribución similar entre hombres y mujeres con un $53 \%$ y $46 \%$ respectivamente.

Al momento de la evaluación, los atletas se encontraban en una etapa precompetitiva dentro de su macrociclo deportivo, con entrenamientos matutinos y vespertinos de lunes a sábado con un promedio de 25 horas de entrenamiento a la semana.

c) Antecedentes de lesiones

Previo a las evaluaciones isocinéticas, fue recabada la información antropométrica (peso, talla, IMC), antecedentes de lesiones musculares en isquiotibiales, características del entrenamiento y la antigüedad practicando el deporte de cada atleta (Tabla 1).

d) Evaluación isocinética

Para este estudio se utilizó el equipo isocinético CSMI Humac Norm. La calibración y las evaluaciones isocinéticas fueron realizadas en todos los casos por un médico, siendo el mismo para todos los casos. Se realizaron 2 
series de 5 repeticiones con 1 minuto de descanso entre series para cada lado, comenzando siempre con el lado dominante de cada atleta. Se utilizó un protocolo concéntrico/concéntrico a $60 \%$ y $120 \%$ s. Previo a cada prueba todos los participantes realizaron un calentamiento en bicicleta ergométrica fija durante 10 minutos (Nerín, Montaño, Carrasco y Martínez, 2005; Santos Silva et al., 2016).

\section{e) Posicionamiento}

Para cada evaluación, los participantes fueron colocados en posición sedente en la mesa de pruebas del equipo CSMI Humac Norm. Para garantizar su posicionamiento y alineación de segmentos, se utilizaron cinchos estabilizadores en la zona de pecho y muslo y se realizó una sujeción del tobillo y pie al brazo de palanca, además de un cojín lumbar para mantener el apoyo del tronco en el respaldo del equipo, logrando un rango de movimiento libre de $0^{\circ}$ de extensión hasta $90^{\circ}$ de flexión de rodilla y un ángulo de $85^{\circ} \mathrm{del}$ muslo en relación con el tronco (Figura 1).

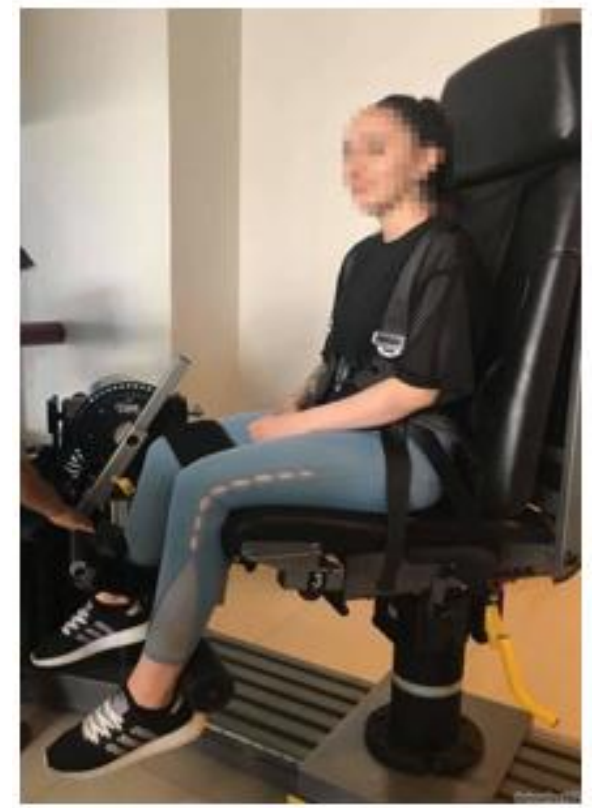

Figura 1. Posicionamiento y alineación de segmentos en la mesa de pruebas.

\section{f) Procedimiento de evaluación}

Para las evaluaciones, el grupo de atletas se dividió en dos partes, los cuales fueron evaluados en dos días distintos aunque a misma hora del día. Previo a la evaluación se les explicó el procedimiento de la prueba y la tarea que debían ejecutar,

Posterior al calentamiento, cada atleta fue colocado en la mesa de pruebas del dinamómetro y fue posicionado debidamente como se mencionó 
anteriormente. Una vez posicionados, se les dio la oportunidad a los atletas de probar el movimiento que tenían que ejecutar durante la prueba de manera activa y sin resistencia para familiarizarlos con el equipo y la prueba. Tras 5 minutos de descanso, se comenzó de la misma manera con la extremidad no dominante.

\section{Resultados}

Se evaluó la fuerza de la musculatura extensora y flexora de rodilla de 15 atletas ( 8 hombres y 7 mujeres) mediante dinamometría isocinética; $\mathrm{Al}$ momento de la evaluación todos los atletas eran mayores de 18 años $(21 \pm 2)$, además, reportaron un promedio de 14 años de antigüedad practicando el deporte. Cabe señalar que todos los participantes presentaron lateralidad homogénea diestra y ninguno de ellos se había sometido previamente a una evaluación isocinética. Las características antropométricas se encuentran en la tabla 1 .

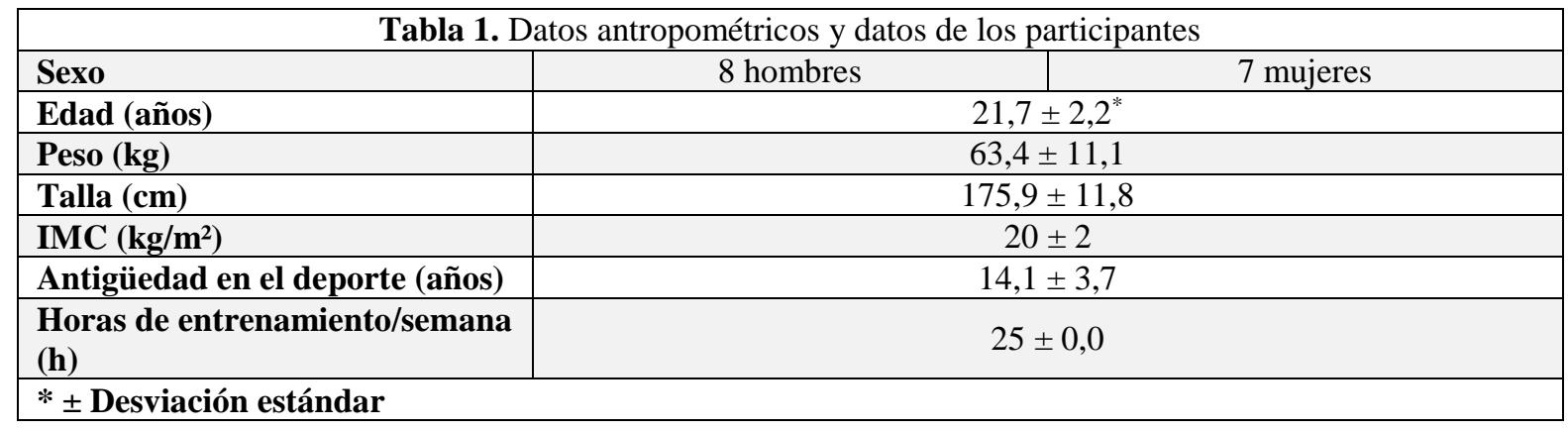

Al comparar los resultados obtenidos tras las evaluaciones isocinéticas, se observó en todos los casos valores superiores en los músculos extensores de rodilla en comparación con los músculos flexores en referencia al pico máximo de torque $(\mathrm{Nm})$, lo cual fue más notorio en la extremidad izquierda a $60 \%$ s (Tabla 2 y 3 ). Así mismo, en promedio, de acuerdo a todos los casos, la extremidad inferior izquierda presentó el mayor desequilibrio muscular entre agonistas y antagonistas con un $57 \%$ a $60 \%$, a diferencia de la extremidad derecha la cual solo presentó un $60 \%$ a la misma velocidad. Como se esperaba, la diferencia entre músculos flexores y extensores de rodilla se vería reducida a mayores velocidades, de este modo, a $120 \%$ s presentaron un desequilibrio de tan solo $65 \%$ y $68 \%$ respectivamente (Tabla 4). 
Tabla 2. Características de la prueba y pico máximo de torque $(\mathrm{Nm})$ a $60 \% \mathrm{~s}$

\begin{tabular}{|l|c|c|c|c|} 
& \multicolumn{2}{c|}{ Músculos flexores } & \multicolumn{2}{c|}{$60^{\circ} / \mathrm{s}$} \\
\hline Velocidad & \multicolumn{2}{|c|}{$60^{\circ} / \mathrm{s}$} & \multicolumn{2}{c|}{$0^{\circ}$ a $90^{\circ}$} \\
\hline Rango de movimiento & \multicolumn{2}{|c|}{$0^{\circ}$ a $90^{\circ}$} & \multicolumn{2}{c|}{5} \\
\hline Número de repeticiones & \multicolumn{2}{|c|}{5} & Derecho & Izquierdo \\
\hline Lado & Derecho & Izquierdo & $173,4 \pm 45$ & $158,6 \pm 38$ \\
\hline $\begin{array}{l}\text { Pico máximo de torque* } \\
\text { (Flexores/extensores) }\end{array}$ & $102,5 \pm 24^{*}$ & $88,4 \pm 29$ & & \\
\hline $\begin{array}{l}\text { *Pico máximo de torque }(\mathrm{Nm}) \\
\text { * Desviación estándar }\end{array}$ & & & \\
\hline
\end{tabular}

Tabla 3. Características de la prueba y pico máximo de torque $(\mathrm{Nm})$ a $120^{\circ} / \mathrm{s}$

\begin{tabular}{|c|c|c|c|c|}
\hline & \multicolumn{2}{|c|}{ Músculos flexores } & \multicolumn{2}{|c|}{ Músculos extensores } \\
\hline Velocidad & \multicolumn{2}{|c|}{$120^{\circ} / \mathrm{s}$} & \multicolumn{2}{|c|}{$120 \% \mathrm{~s}$} \\
\hline Rango de movimiento & \multicolumn{2}{|c|}{$0^{\circ}$ a $90^{\circ}$} & \multicolumn{2}{|c|}{$0^{\circ}$ a $90^{\circ}$} \\
\hline Número de repeticiones & \multicolumn{2}{|c|}{5} & \multicolumn{2}{|c|}{5} \\
\hline Lado & Derecho & Izquierdo & Derecho & Izquierdo \\
\hline $\begin{array}{l}\text { Pico máximo de torque* } \\
\text { (Flexores/extensores) }\end{array}$ & $92,9 \pm 25^{*}$ & $87,9 \pm 40$ & $139,4 \pm 45$ & $123,5 \pm 37$ \\
\hline
\end{tabular}

\begin{tabular}{|c|c|c|}
\hline & $60^{\circ} / \mathrm{s}$ & $120^{\circ} / \mathrm{s}$ \\
\hline Lado derecho & $60 \pm 7 *$ & $68 \pm 9$ \\
\hline Lado izquierdo & $57 \pm 9$ & $65 \pm 8$ \\
\hline
\end{tabular}

El $46 \%$ de los atletas presentó alguna lesión muscular en los isquiotibiales en el último año, del mismo modo, el $46 \%$ de estos presentó un desequilibrio muscular significativo en al menos una extremidad. Cabe mencionar que en todos los casos de lesión muscular coincidió con la extremidad cuyo desequilibrio muscular era significativo. Además, en dos casos de desequilibrio muscular no se había presentado aún ninguna lesión en isquiotibiales así como también, un participante presentó desequilibrio muscular en ambas extremidades, sin embargo, solo había sufrido de lesión muscular en una sola extremidad.

Para establecer la relación entre las lesiones musculares de isquiotibiales y el desequilibrio muscular entre flexores y extensores de rodilla, se utilizó la prueba exacta de Fisher como prueba para analizar datos categóricos y examinar la asociación, comúnmente utilizada con tamaños de muestra pequeños. De esta manera se encontró significancia estadística en aquellos casos con un desequilibrio muscular significativo que habían padecido una lesión muscular en isquiotibiales $(\mathrm{p}=.007)$, los cuales 
corresponden al $47 \%$ de la muestra. El $40 \%$ representaba a aquellas personas sin desequilibrio muscular y sin lesión de isquiotibiales, y el $13 \%$ restante a aquellas personas que tienen un desequilibrio muscular significativo pero que aún no habían sufrido una lesión muscular (Tabla 5).

\begin{tabular}{|l|c|c|}
\hline \multicolumn{3}{|c|}{ Tabla 5. Casos de desequilibrio y lesión muscular } \\
\hline Total de participantes & Número de casos & Asociación \\
\hline $\begin{array}{l}\text { Casos de desequilibrio } \\
\text { muscular }\end{array}$ & 15 & \\
\hline Casos de lesión muscular & 9 & $\mathrm{P}=.007$ \\
\hline $\begin{array}{l}\text { Casos de desequilibrio } \\
\text { muscular con lesiones } \\
\text { muscular }\end{array}$ & 7 & \\
\hline
\end{tabular}

\section{Discusión}

Dentro de los datos obtenidos se encontraron coincidencias con autores como Croisier et al. (2008), Cameron, Adams y Maher (2003) y Orchard (2001) quienes han encontrado la misma relación entre estas dos variables en diversos deportes, así mismo, el desequilibrio entre estos grupos musculares también se ha asociado con otro tipo de lesiones, entre las que destacan las lesiones del ligamento cruzado anterior (Cheung, Smith y Wong, 2012; Santos et al., 2016). Las mediciones a través de dinamometría isocinética han sido utilizadas como valor predictivo, tratamiento y seguimiento y valoración (Cruz et al., 2008), por ser un método preciso que proporciona información fiable y manejable. Tras la evaluación, se encontraron diferencias significativas entre la magnitud física de pico máximo de torque $(\mathrm{Nm})$ en el aparato extensor en comparación con el aparato flexor de rodilla, probablemente debido al mayor requerimiento físico para ejecutar el gesto motor predominante en el taekwondo como saltos y lanzamientos de patadas (Martínez et al., 2014), así mismo, contrario a lo que mencionan Ferreira, Gomes, Santos, de Arruda y de Franca (2010), la lateralidad homogénea derecha en el grupo muestra, pudo condicionar el mayor desequilibrio muscular encontrado en la extremidad inferior izquierda, como también, los menores valores de pico máximo de torque generados en esa misma extremidad.

Estudios previos han propuesto la idea de una falsa debilidad de isquiotibiales a la hora de medir la relación isquiotibiales: cuádriceps $(\mathrm{H}: \mathrm{Q}$ por sus siglas en inglés) asociada a un desarrollo mayor de la fuerza del cuádriceps que a una debilidad propiamente dicha de los isquiotibiales, lo cual puede tener lugar en aquellas disciplinas donde predominen actividades de salto y golpeo con la extremidad inferior (Bogdanis y Kalapotarakos, 2016; Cameron et al., 2003), por otro lado, se ha estudiado también la mayor activación neuromuscular de cuádriceps en comparación con los isquiotibiales 
en las acciones de recepción del salto y/o cambios de dirección y aceleración (Vanmeerhaeghe y Romero, 2013).

Así mismo, se pudo identificar mayor variación en la relación $\mathrm{H}$ : Q a velocidades más bajas, es decir, $60 \%$ s, misma que, como se esperaba, se vería reducida a mayor velocidad (Rosone, Fogarty y Mahaffey, 2001). En dos casos particulares de desequilibrio muscular no se había presentado ninguna lesión muscular de isquiotibiales en el último año, si bien, autores han señalado al desequilibrio muscular como factor de riesgo y predictor de lesiones, otros señalan a este tipo de lesiones como multifactoriales y a pesar de que existan factores predisponentes es necesaria la presencia de un factor detonante (Balius et al., 2013).

Si bien, se pudo identificar un desequilibrio muscular en el $46 \%$ de los atletas evaluados, para calcular dicho desequilibrio entre el cuádriceps e isquiotibiales se utilizó el índice convencional. Autores como Ayala et al. (2011) señalan esta modalidad como método idóneo para evaluar el desequilibrio, sin embargo, otros autores como Aagaard et al. (1998) sugieren realizar las evaluaciones con contracciones excéntrico/concéntrico (índice funcional) para isquiotibiales y cuádriceps respectivamente, apoyándolo en que la actividad muscular que ejecutan los isquiotibiales durante los gestos en el que el cuádriceps se contrae concéntricamente, es distinta y más bien se trata de una contracción excéntrica. A pesar de contar con estas dos posturas aún no se ha llegado a un consenso universal al respecto.

\section{Conclusión}

La alta incidencia de lesiones musculares que suelen presentarse en el ámbito deportivo y específicamente en esta población debe ser, en la medida de lo posible, prevenida para evitar las consecuencias y complicaciones que estas conllevan. Ha sido ampliamente estudiada la relación entre el desequilibrio muscular entre flexores y extensores de rodilla y las lesiones musculares en otros deportes, sin embargo, hasta la fecha existen pocos estudios enfocados específicamente en el taekwondo, por lo que son necesarios más estudios que examinen diferentes variables específicas a esta disciplina.

Es prioritaria la intervención multidisciplinaria de profesionales entorno al atleta de alto rendimiento en la implementación de evaluaciones y test enfocados a detectar datos de alarma que pudieran predisponer al atleta a desarrollar alguna lesión deportiva en el futuro y que esta pueda ser atendida de manera temprana y en última instancia mejorar su desempeño deportivo.

Por otro lado es importante destacar la necesidad de investigar sobre nuevos protocolos para establecer valores estándar en este tipo de procedimientos de evaluación y plantear métodos más apropiados de 
evaluación de acuerdo a la actividad muscular que se desarrolla en una actividad funcional o en un determinado gesto deportivo.

\section{References:}

1. Aagaard, P., Simonsen, E. B., Magnusson, S. P., Larsson, B., \& DyhrePoulsen, P. (1998). A new concept for isokinetic hamstring: quadriceps muscle strength ratio. The American journal of sports medicine, 26(2), 231-237. https://doi.org/10.1177/03635465980260021201.

2. Altarriba-Bartes, A., Drobnic, F., Til, L., Malliaropoulos, N., Montoro, JB e Irurtia, A. (2014). Epidemiología de las lesiones en atletas de taekwondo de élite: estudio retrospectivo transversal de dos períodos olímpicos. BMJ abierto, 4 (2), e004605. doi: 10.1136 / bmjopen2013-004605.

3. Ayala, F., Sainz de Baranda, P., de Ste Croix, M. y Santonja, F. (2012). Validez y fiabilidad de los ratios de fuerza isocinética para la modificación de desequilibrios musculares. [en línea] apunts.org. Disponible en: https://www.apunts.org/es-validezfiabilidad-ratios-fuerza-isocinetica-articulo-X0213371712807039 [Consultado el 19 de febrero de 2020].

4. Balius, R. y Pedret, C. (2013). Lesiones Musculares en el Deporte (1. ${ }^{\mathrm{a}}$ ed., pp. 27-36). Editorial Médica Panamericana.

5. Muscle Injuries Clinical Guide 3.0, Guía de manejo de lesiones musculares: Guía de práctica clínica. FCBarcelona, 2015.

6. Bogdanis, G. C., \& Kalapotharakos, V. I. (2016). Knee Extension Strength and Hamstrings-to-Quadriceps Imbalances in Elite Soccer Players. International journal of sports medicine, 37(2), 119-124. https://doi.org/10.1055/s-0035-1559686.

7. Cameron, M., Adams, R. and Maher, C., (2003). Motor control and strength as predictors of hamstring injury in elite players of Australian football. Physical Therapy in Sport, [online] 4(4), pp.159-166. Available <https://www.sciencedirect.com/science/article/abs/pii/S1466853X03 000531> [Accessed 16 August 2020].

8. Croisier, J.-L., Ganteaume, S., Binet, J., Genty, M., \& Ferret, J.-M. (2008). Strength Imbalances and Prevention of Hamstring Injury in Professional Soccer Players: A Prospective Study. The American Journal of Sports Medicine, 36(8), 14691475. https://doi.org/10.1177/0363546508316764.

9. Cruz-Medina, Eva y León-Hernández, Saúl Renán, y ArellanoHernández, Aurelia y Martínez-Gonzaga, Enrique y García-Guerrero, Eduardo y Coronado-Zarco, Roberto (2008). Evaluación isocinética y estado funcional en pacientes posoperados por hernia de disco lumbar. 
Cirugía y Cirujanos, 76 (5), 373-380. [Fecha de Consulta 29 de Enero de 2021]. ISSN: 0009-7411. Disponible en: https://www.redalyc.org/articulo.oa?id=662/66276503

10. Cheung, R., Smith, A., \& Wong, D. (2012). H:Q Ratios and Bilateral Leg Strength in College Field and Court Sports Players, Journal of Human Kinetics, 33(2012),

63-71. doi: https://doi.org/10.2478/v10078-012-0045-1.

11. Cruz-Medina, Eva y León-Hernández, Saúl Renán y ArellanoHernández, Aurelia y Martínez-Gonzaga, Enrique y García-Guerrero, Eduardo y Coronado-Zarco, Roberto (2008). Evaluación isocinética y estado funcional en pacientes posoperados por hernia de disco lumbar. Cirugía y Cirujanos, 76 (5), 373-380. [Fecha de Consulta 15 de Agosto de 2020]. ISSN: 0009-7411. Disponible en: https://www.redalyc.org/articulo.oa?id=662/66276503.

12. Ferreira, Aparecido Pimentel, Gomes, Sérgio Adriano, Ferreira, Carlos Ernesto Santos, Arruda, Miguel de, \& França, Nanci Maria de. (2010). Avaliação do desempenho isocinético da musculatura extensora e flexora do joelho de atletas de futsal em membro dominante e não dominante. Revista Brasileira de Ciências do Esporte, 32(1), 229243. https://doi.org/10.1590/S0101-32892010000400016.

13. Fortina, M., Mangano, S., Carta, S. \& Carulli, C.. (2017 agosto 4). Analysis of Injuries and Risk Factors in Taekwondo during the 2014 Italian University Championship. Joints, v.5, 168-172. 2019, Noviembre 7, De PubMed. Base de datos.

14. Fort Vanmeerhaeghe, A. y Romero Rodriguez, D. (2013). Análisis de los factores de riesgo neuromusculares de las lesiones deportivas. [en línea] apunts.org. Disponible en: https://www.apunts.org/es-analisisfactores-riesgo-neuromusculares-lesiones-articuloX0213371713445417 [Consultado el 19 de febrero de 2020].

15. de Hoyo, M., Naranjo-Orellana, J., Carrasco, L., Sañudo, B., JiménezBarroca, J. J., \& Domínguez-Cobo, S.. (2013). Revisión sobre la lesión de la musculatura isquiotibial en el deporte: factores de riesgo y estrategias para su prevención. Revista Andaluza de Medicina del Deporte, 6(1), 30-37. Recuperado en 08 de noviembre de 2019, de http://scielo.isciii.es/scielo.php?script=sci_arttext\&pid=S1888$75462013000100007 \& \operatorname{lng}=\mathrm{es} \&$ tlng=es.

16. Loeza, P., Fritzel, W. and Barrios, J. (2016). Correlación entre prueba isocinética y test de potencia anaeróbica y agilidad. Revista mexicana de investigación en cultura física y deporte, [online] (9), pp.47-59. Available at: http://ened.conade.gob.mx/Documentos/REVISTA\%20ENED/Revist a9/Revista\%20ENED_v2.pdf [Accessed 17 Jan. 2020]. 
17. Maly, T., Mala, L., Bujnovsky, D., Hank, M. y Zahalka, F. (2019). Diferencias morfológicas e isocinéticas de fuerza: variación bilateral e ipsolateral según la actividad deportiva. Medicina abierta (Varsovia, Polonia) , 14 , 207-216. doi: 10.1515 / med-2019-0014.

18. Martínez, L., Pegueros, A., Ortíz, A., Del villar, A., Flores, V. and Pineda, C. (2014). Valoración isocinética de la fuerza y balance muscular del aparato extensor y flexor de la rodilla en taekwondo. Gaceta Médica de México, [online] (3), pp.272-280. Available https://www.anmm.org.mx/GMM/2014/s3/GMM_150_2014_S3_272 -278.pdf [Accessed 4 Dec. 2019].

19. Orchard J. W. (2001). Intrinsic and extrinsic risk factors for muscle strains in Australian football. The American journal of sports medicine, 29(3), 300-303. https://doi.org/10.1177/03635465010290030801.

20. Rosene, JM, Fogarty, TD y Mahaffey, BL (2001). Isquiotibiales isocinéticos: proporciones de cuádriceps en atletas interuniversitarios. Revista de entrenamiento atlético, 36 (4), 378383.

21. Santos, P., Pedrinelli, A., Rubio, E., Guilherme, C. and D’Andrea, J., (2020). Evaluación isocinética de músculos flexores y extensores en jugadores de fútbol profesional antes de iniciar la fase de pretemporada. Revista Latinoamericana de Cirugía Ortopédica, [online] (1), pp.54-57. Available at: $<$ https://www.sciencedirect.com/science/article/pii/S2444972516300 249> [Accessed 26 March 2020].

22. Tlatoa, H. (2014). Torque máximo absoluto e índice convencional isocinético de rodilla en futbolistas profesionales del 2007 al 2012. [en línea] Elsevier.es. Disponible en: https://www.elsevier.es/es-revistarevista-medicina-e-investigacion-353-articulo-torque-maximoabsoluto-e-indice-X2214310614603822 [Consultado el 20 de febrero de 2020].

23. van Dyk, N., Bahr, R., Whiteley, R., Tol, J. L., Kumar, B. D., Hamilton, B., Witvrouw, E. (2016). Hamstring and Quadriceps Isokinetic Strength Deficits Are Weak Risk Factors for Hamstring Strain Injuries: A 4-Year Cohort Study. The American Journal of Sports Medicine, 44(7), 1789 1795. https://doi.org/10.1177/0363546516632526. 